\title{
On the Computational Power of Neural Microcircuit Models: Pointers to the Literature
}

\author{
Wolfgang Maass \\ Institute for Theoretical Computer Science \\ Technische Universität Graz \\ A-8010 Graz, Austria \\ maass@igi.tu-graz.ac.at \\ http://www.igi.tugraz.at/maass
}

\begin{abstract}
This paper provides references for my invited talk on the computational power of neural microcircuit models.
\end{abstract}

Biological neural microcircuits are highly recurrent and consist of heterogeneous types of neurons and synapses, which are each endowed with an individual complex dynamics [4], 6], 3], [7, [8], [23], [20]. Hence neural microcircuits are as different as one can imagine from the familiar boolean circuits in our current generation of computers, but also very different from common artificial neural network models. This has given rise to the question how neural microcircuits can be used for purposeful computations.

There are two quite different ways of approaching this question. One way is to construct circuits consisting of biologically realistic components that can simulate other models for general-purpose computers such as Turing machines [13] or general-purpose artificial neural network models [14, 16], or to construct circuits that carry out specific computations such as for example simpified speech recognition [10]. Another way, which is discussed in my talk, is to recruit with the help of suitable adaptive mechanisms - biologically realistic "found" or emerging models for neural microcircuits for purposeful computations. An inspiring first example for this approach is given in [2]. It became the basis of our new approach towards real-time computing in neural systems in [17. The underlying computational theory is presented in [15], and discussed from a biological point of view in 19. Particular computational consequences of the high dimensionality of neural microcircuits are discussed in [9]. It turns out that this approach yields superior performance in terms of noise robustness, computing speed, and size compared with special-purpose neural circuits that have been constructed by hand for a specific computational task 18. Publicly available software for generating and simulating generic neural microcircuit models, and for evaluating their computational power, is discussed in [21].

Herbert Jaeger discovered independently quite similar phenomena of temporal integration in recurrent circuits in the context of artificial neural network models [1].

Current work addresses the application of the resulting new principles for neural computation to online processing of real-world time-varying inputs, such 
as movement prediction for visual inputs [12, speech recognition in real-time, and real-time processing of sensory inputs on a robot. Another line of current research explores the computational role of specific details of biological neural microcircuits, and the role of learning principles in this context.

\section{References}

1. Auer, P., Burgsteiner, H., and Maass, W. (2002). Reducing communication for distributed learning in neural networks. In Proc. ICANN'2002. Online available as \# 127 on http://www.igi.tugraz.at/maass/publications.html.

2. Buonomano, D.V., and Merzenich, M.M. (1995). Temporal information transformed into spatial code by a neural network with realistic properties. Science 267, 1028-1030.

3. Braitenberg, V., and Schuez, A. (1998). Cortex: Statistics and Geometry of Neuronal Connectivity, 2nd ed., Springer Verlag, Berlin.

4. Cajal, S. Ramón y (1911). Histologie du système nerveux de l'homme et des vertébrés, translated by L. Azoulay. Consejo superior de investigaciones cientificas. Instituto Ramon y Cajal, Madrid, edn. 1972.

5. Douglas, R., and Martin, K. (1998). Neocortex. In The Synaptic Organization of the Brain, G.M. Shepherd, Ed. (Oxford University Press), 459-509.

6. De Felipe, J. (1993). Neocortical neuronal diversity: chemical heterogeneity revealed by colocalization studies of classic neurotransmitters, neuropeptides, calcium-binding proteins, and cell surface molecules. Cerebral Cortex 7, 476-486.

7. Gupta, A., Wang, Y., and Markram, H. (2000). Organizing principles for a diversity of GABAergic interneurons and synapses in the neocortex. Science 287, 273-278.

8. Gupta, A., Silberberg, G., Toledo-Rodriguez, M., Wu, C.Z., Wang, Y., and Markram, H. (2002). Organizing principles of neocortical microcircuits. Cellular and Molecular Life Sciences, in press.

9. Häusler, S., Markram, H., and Maass, W. (2002). Low dimensional readout from high dimensional neural circuits, submitted for publication. Online available as \# 137 on http://www.igi.tugraz.at/maass/publications.html.

10. Hopfield, J.J., and Brody, C.D. (2001). What is a moment? Transient synchrony as a collective mechanism for spatio-temporal integration. Proc. Natl. Acad. Sci., USA, 89(3), 1282.

11. Jaeger, H. (2001). The "echo state" approach to analyzing and training recurrent neural networks, submitted for publication.

12. Legenstein, R.A., Markram, H., and Maass, W. (2002). Input prediction and autonomous movement analysis in recurrent circuits of spiking neurons, submitted for publication. Online available as \# 140 on http://www.igi.tugraz.at/maass/publications.html.

13. Maass, W. (1996). Lower bounds for the computational power of networks of spiking neurons. Neural Computation 8(1):1-40. Online available as \# 75 on http://www.igi.tugraz.at/maass/publications.html.

14. Maass, W. (1997). Fast sigmoidal networks via spiking neurons. Neural Computation 9:279-304. Online available as \# 82 on http://www.igi.tugraz.at/maass/publications.html.

15. Maass, W., and Markram, H. (2002). On the computational power of recurrent circuits of spiking neurons, submitted for publication. Online available as \# 135 on http://www.igi.tugraz.at/maass/publications.html. 
16. Maass, W., and Natschläger, T. (1997). Networks of spiking neurons can emulate arbitrary Hopfield nets in temporal coding. Network: Computation in Neural Systems 8(4):355-372. Online available as \# 93 on http://www.igi.tugraz.at/maass/publications.html.

17. Maass, W., Natschläger, T., and Markram, H. (2002). Real-time computing without stable states: A new framework for neural computation based on perturbations. Neural Computation, in press. Online available as \# 130 on http://www.igi.tugraz.at/maass/publications.html.

18. Maass, W., Natschläger, T., and Markram, H. (2002a). Real-time computing with emergent neural microcircuit models, submitted for publication.

19. Markram, H., Ofer, M., Natschläger, T., and Maass, W. (2002) Temporal integration in neocortical microcircuits. Cerebral Cortex, in press. Online available as \# 142 on http://www.igi.tugraz.at/maass/publications.html

20. Mountcastle, V.B. (1998). Perceptual Neuroscience: The Cerebral Cortex, Harvard University Press (Cambridge).

21. Natschläger, T., Markram, H., and Maass, W. (2002). Computer models and analysis tools for neural microcircuit models. In A Practical Guide to Neuroscience Databases and Associated Tools, R. Kötter, Ed., Kluver Academic Publishers (Boston), in press.

22. Shepherd, G.M. (1988). A basic circuit for cortical organization. In Perspectives in Memory Research, M. Gazzaniga, Ed., MIT-Press, 93-134.

23. Thomson, A., West, D.C., Wang, Y., and Bannister, A.P. (2002). Synaptic connections and small circuits involving excitatory and inhibitory neurons in layers 2 to 5 of adult rat and cat neocortex: triple intracellular recordings and biocytin-labelling in vitro. Cerebral Cortex, in press. 